here on the instruments which might be used on such facilities. Ultracold and very cold neutrons (UCN $\sim 500 \AA$; VCN $\sim 80 \AA$ ) may be more available in the future, even as polarized beams, extending the neutron wavelength range beyond anything available in the past; more food for thought.

The spin-echo technique is highly regarded for futuristic instrumentation, and this is given a brief review. The developments of film methods of recording neutron scattering are most exciting and should stimulate new science.

The IAEA meetings constitute the best forum for the discussion and dissemination of new techniques in neutron scattering, and this section is no exception; indeed it is probably true to say that this work is more healthy now than ever before.

Sessions III \& IV. This extensive section contains many papers concerned with rotation or hindered rotation of groups such as methyl and ammonium groups, though there are a few papers rather out of place. As this is a discipline for which there is no obvious single international journal for such papers, this collection will be found most useful to those studying this field which ranges over plastic crystals in the main. At the previous IAEA meeting this field was open and clear; we are now getting to grips with the new problems raised and the near future will certainly see considerable developments. Unfortunately there is no article which gazes into the crystal ball!

Session V. The novel experiments on liquid crystals here reported have a close affinity with those on plastic crystals, and it is good editorial management to get this session in the same volume as the previous two. The coverage of polymer work is probably not so complete as that for liquid and plastic crystals but is nevertheless valuable.

Session VI. Further progress on non-superfluid and superfluid ${ }^{4} \mathrm{He}$ is reported, and the review touches also on ${ }^{3} \mathrm{He}$, though detailed experimental results on this system are not reported. Other liquids studied are argon and lead, but there must be much other work not covered in these volumes.

Sessions VII \& VIII. These sessions begin with a review of incommensurate structural phase transitions, phenomena which should be noted by all crystallographers. These may be much more common than previously expected, and the crystallographer would do well to call on all his experience of the many structural studies and remember those cases where the Bragg peaks showed the modulation characteristic of the incommensurate phase.

Most of the contributed papers are concerned with spin waves, though there is the occasional phonon paper. As phonons were the subject of a conference in Paris in September 1977, such papers in this volume are inconvenient. For full coverage the Proceedings of the International Conference in Lattice Dynamics, Paris, 5-9 September 1979 [(1978), edited by M. Balkanski. Paris: Flammarion] should be consulted.

Session IX. Hydrogen in metals: diffusion, dispersion curves and structure all need the neutron scattering technique, and the current work in the field is well covered in this volume.

Session X. Structure plays a more dominant role in this final session than elsewhere in these volumes, but efforts are now being extended to study the dynamics of these systems, typified by nitrogen or methane on exfoliated graphite. This work seems to be in need of the crystallographer's skill in understanding structure; such an understanding is essential for the interpretation of the inelastic scattering. This section is as complete a coverage as one could hope for in one place, though it may soon be out of date.

These volumes will become just as important as the previous volume from the Grenoble meeting in 1972. No group which aspires to neutron scattering should be without their copy, and the price should be compared with that of any specialized journal. There is an advantage over a journal as each paper is followed by a record of the discussion which was generated at the time of presentation.

Our poor knowledge of the structure of plastic and liquid crystals and of surfaces may prompt crystallographers to deviate from their present course of research, maybe even applying X-ray scattering to these self-same systems. Of course no comparison of the value of neutron scattering with $\mathrm{X}$-ray scattering could be expected in these volumes, but the clear progress in the former activity should stimulate advances in the latter.

Department of Physics

G. S. PAWLEY

University of Edinburgh

Edinburgh EH9 $3 \mathrm{JZ}$

Scotland

Acta Cryst. (1979). A35, 349-350

Electron diffraction 1927-1977 (Conference Series No. 41). Edited by P. J. Dobson, J. B. Pendry and C. J. Humphreys. Pp. xi +442 . The Institute of Physics, Bristol and London, 1978. Price $£ 25 \cdot 00$, US $\$ 49.00$.

This volume, with 67 papers in total, consists of invited and contributed papers which were presented at the International Conference on Electron Diffraction held at Imperial College, London University, from 19 to 21 September 1977. As this conference was planned in celebration of the 50th Anniversary of the discovery of electron diffraction in 1927 , it is natural that the book is entitled 'Electron Diffraction 1927-1977', although it contains little historical material. In the half-century since its discovery, electron diffraction has played an important and unique role in the study of the structure of matter, but it is also true that it has been accompanied by inherent theoretical difficulties due to the complexity of the process of electron scattering by matter. However, this complexity may be more informative eventually, and may continue to be an inexhaustible source of attractive research problems worth pursuing further. In the last decade too, stimulating discoveries of diffraction effects, such as the critical-voltage effect and electron channelling, have been made, and advances in the theory of LEED as well as in the theory and application of electron-microscopic lattice images have been considerable. And, relating to all of these topics, the dynamical theory of electron diffraction has been explored very extensively from various approaches. In these respects, the publication of this book is quite timely.

The text consists of seven chapters: 1. Dynamical theory; 2. Diffuse scattering; 3 . Structure factor and symmetry determination; 4. Low energy electron diffraction; 5. Instrumentation; 6. Defect structure; 7. Miscellaneous. Each chapter, except Chapter 7, contains one or more invited 
papers, each giving a survey of the topic of relevance. In Chapter 1 , Howie points out the central themes of the dynamical theory, related to, e.g., the optical potential, the cross-over of the dispersion surface, and various calculation techniques. This chapter includes many papers on the critical-voltage effect as well as the phenomenon of electron channelling. It is remarkable that the dynamical theory has been successfully applied to the channelling effect of electrons of energy as high as $15 \mathrm{MeV}$ (Worm et al.). In Chapter 2, dealing with short-range order diffuse scattering, Watanabe describes a number of examples of diffuse scattering reflecting the form of the Fermi surface for some disordered alloys. As described by Goodman in Chapter 3, accurate measurement of structure factors and the determination of crystal symmetries are two of the most beautiful features of recent electron diffraction research. It is interesting to see that the accurate structure factors thus becoming available may be useful, e.g. in detecting the solidstate bonding effect (Smart \& Humphreys). In the same chapter, Cowley emphasizes the important and unique capability of electron diffraction in crystal structure determination, especially for extremely small samples. In this connection, he also points out a number of difficulties to be overcome in order to realize the full potential of electron diffraction. Incidentally, this chapter also contains a few papers relating to electron channelling.

Pendry, reviewing recent advances in LEED in Chapter 4, gives a compact summary of the layer methods that are now current in LEED, and also discusses the relativistic effects in LEED which become significant for heavier atoms through spin-orbital coupling. He also mentions the impact of LEED theory on neighbouring problems such as EXAFS. The same chapter contains two experimental papers dealing with spinpolarized LEED (Müller et al., Unertl et al.), which suggest a fruitful area of electron diffraction study for the future. The paper by Mulvey in Chapter 5, being a review of the development of instrumentation for electron diffraction, is of special interest as it reminds us of historical experiments performed by Davisson \& Germer, Thomson \& Reid, Kikuchi and others. Chapter 6 is headed by a review paper by Hirsch. It is worth mentioning that two papers in the final chapter deal with liquid structure.

Besides the papers briefly referred to in the above, readers will find many other attractive papers in this volume which are equally to be appreciated. Scientific standards of the papers compiled are uniform and generally high. In this volume, readers have a useful survey of the important aspects, if not the whole, of the recent advances in electron diffraction research.

Shizuo MiYaKe

\section{Department of Physics}

Faculty of Science and Technology

Science University of Tokyo

Noda City

Chiba 278

Japan
Acta Cryst. (1979). A35, 350

\section{Books Received}

The following books have been received by the Editor. Brief and generally uncritical notices are given of works of marginal crystallographic interest; occasionally a book of fundamental interest is included under this heading because of difficulty in finding a suitable reviewer without great delay.

Кристаллография. Ву М. Р. Шасколъская (Crystallography. By M. P. Shaskol'SkAYA). Pp. 391. Vysshaya Shkola publishing house, Moscow, 1976. Price 1 r. 52 k. A review of this book, by $Z$. Kaluski, has been published in the February 1979 issue of Journal of Applied Crystallography, pages 139-140.

Environment international. A journal of science, technology, health, monitoring and policy. Editors, A. A. MogHIssI and B. D. Moghissi. Pergamon Press. Vol. 1 (1978) subscription, US\$72.60 p.a.

The harvest of a quiet eye. A selection of scientific quotations. By A. L. MACKAY. Pp. xii +192 . The Institute of Physics, Bristol and London. Price $£ 5 \cdot 20$.

Elements of $\mathbf{X}$-ray diffraction (2nd edition). By B. D. Cullrty. Pp. xii + 555. Addison-Wesley, 1978. Price US $\$ 21.95, £ 16 \cdot 50$. This book was first published two decades ago in 1956. Its production now, as a second edition, is a measure of its success and usefulness.

It is a pity that the title of this book is not more explicit, for it is in fact addressed rather narrowly to the problems of materials science and technology, and of metallurgy in particular. A more correct title would have been 'Elements of $X$-ray Diffraction for Students of Metallurgy'.

It is also a pity that students of metallurgy are given so poor a glimpse of what X-ray diffraction achieves in the way of structure determination. Only 20 pages are allotted to crystal structure determination and, in this section, the one example that is given is a zinc blende structure solved from a powder pattern whose intensities are listed in terms of '...$s$, $w, v s$...' giving a mildly antiquarian atmosphere. However, apart from this one important area, it must be said that the coverage of the book is impressively comprehensive.

The first edition of this book was reviewed in this journal in 1957 [Preston, G. D. (1957) Acta Cryst. 10, 389]. Virtually all that was said then remains true of this second edition, except that quite a large number of new techniques, such as energy-dispersive analysis, time-analysis diffractometry, the Auger effect, and so on, have been brought in, giving this edition a highly up-to-date flavour - for metallurgical applications. 